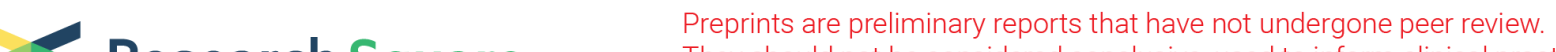 Research Square They should not be considered conclusive, used to inform clinical practice, or referenced by the media as validated information.
}

\section{BMSCs Seeding in Different Scaffold Incorporation with Hyperbaric Oxygen Treat Seawater Immersed Bony Defect}

gan zhang ( zhanggan10282715@163.com )

the 901th Hospital of Chinese People's Liberation Army

\section{Xiaosong Chen}

the 901 th Hospital of the

\section{Xunsheng Cheng}

The 901th Hosptial of the Joint Logistics Support Force of the Chinese People's Liberation Army

\section{Xiuwu Ma}

The 901th Hospital of Chinese People's Liberation Army

\section{Congcong Chen}

The 901th Hospital of Chinese People's Liberation Army

\section{Research article}

Keywords: BMSCs, HBO, seawater immersed bone defect, n-HA, $\beta-T C P, P L G A$

Posted Date: November 2nd, 2020

DOI: https://doi.org/10.21203/rs.3.rs-44123/v2

License: (c) (1) This work is licensed under a Creative Commons Attribution 4.0 International License.

Read Full License

Version of Record: A version of this preprint was published at Journal of Orthopaedic Surgery and Research on April 13th, 2021. See the published version at https://doi.org/10.1186/s13018-021-02368-8. 


\section{Abstract}

Introduction: The experiment was undertaken to estimate the effect of BMSCs seeding in different scaffold incorporation with HBO on the repair of seawater immersed bone defect. And future compared nHA/PLGA with $\beta$-TCP/PLGA as scaffold in treatment effect of seawater immersed bone defect.

Methods: 60 New Zealand White rabbits with standard seawater defect in radius were randomly divided to group A (implant with nothing), group B (implanted with atuogenous bone), group C (implanted with nHA/PLGA/BMSCs, and Group D (implanted with $\beta$-TCP/PLGA/BMSCs). After implant, each rabbit receive HBO treatment at 2.4 ATA $100 \%$ oxygen for 120 minutes per day for 2 weeeks. Radiograph, histological and biomechanical examination were used to analyze osteogenesis.

Result: X-ray analysis show that n-HA/PLGA/BMSCs and $\beta$-TCP/PLGA/BMSCs could accelerate the new bone formation, and the new bone formation in group $C$ was lager than in group $D$ or group $A$, and close to group $B(P<0.05)$. After 12 weeks, in group $A$, defect without scaffold show a loose connect tissue filled in the areas. The medullary canal in group $B$ was recanalizated. Defect in group $C$ and $D$ show a larger number of wove bone formation. The new wove bone formation in defect areas in group $C$ was lager than $D$. The mechanical examination revealed ultimate strength at 12 weeks were group $D>$ group $C>$ group $B>$ group $A(P<0.05)$.

Conclusion: Scaffold of n-HA/PLGA and $\beta$-TCP/PLGA incorporation with HBO and BMSCs were effective to treat seawater immersed bone defect, and n-HA/PLGA was more excellent than $\beta$-TCP/PLGA.

\section{Introduction}

Healing bone defects was a difficult problem in orthopaedics. when bone defect was immersed by seawater, the repairing become more difficult. Autologous graft and allograft were possible meothods. Autologous bone graft is regarded as gold standard in treating bone defect. However, the donor site bone source is limited, obtaining the autologenous bone was an invasive operation, which can lead to high donor site morbidity ${ }^{1-2}$. Allograft have a potential of transmission of diseases and leading to immune response. Bone tissue engineering was a promising method that can overcome these problems mentioned above.

PLGA was widely used in bone tissue engineering due to its nontoxic and biodegrade as a scaffold. PLGA has been approved by Food and Drug Administration for human clinical appliations ${ }^{3}$. But it has poor mechanical strength and cell affinity which limits its using in bone tissue engineering ${ }^{4-6}$.In addition the acidic degradation can lead to inflammatory reation ${ }^{7-8}$. McBane JE reported that PLGA films were implanted subcutaneously in a rodent model which caused acute inflammatory response ${ }^{9}$. PLGA scaffold has been successfully repaired bone defect in $\mathrm{SD}^{10}$. However, PLGA isn't an excellent scaffold in bone tissue engineering owing to its hydrophobicity and lack of bioactive properties and its degradation 
products leading to inflammation. Its properties can be improved by combinating with other matericals. In our study there are two matericals to be try:(1)n-HA,(2) $\beta$-TCP.

HBO theray is that the patients expose to $100 \%$ oxygen under elevated pressure. Ahmed Jan,et al ${ }^{11}$ evaluated autogenous bone graft and autogenous bone graft with HBO by Micro-CT analysis and histomorphometric analysis and found that new bone formation was more in autogenous bone graft with $\mathrm{HBO}$, suggesting that $\mathrm{HBO}$ can enable the bony healing of critical-sized bone defect. HBO therapy is thought to increase healing by increasing the amout of oxygen dissolved in blood. J.P. Grassmann et al. ${ }^{12}$ HBO therapy enhance bone repairing, which may attribute to an increase in angiogenesis.

\section{Material And Methods}

\section{Experimental main material and reagents}

BMSCs, MSC growth medium, trypsin were purchased from Cyagen Biosciences Inc(Guang dong, China). The $n$-HA/PLGA and $\beta$-TCP/PLGA scaffold ( $4 \mathrm{~mm} \times 15 \mathrm{~mm}$, cylinder) was provided by The Shandong Province key Laboratory of Medical Polymer Materials(Ji nan,China).

\section{Cell culture}

BMSC were obtained from cyagen at passage 2. The BMSCs were were shawed and cultured in growth medium from cyagen in humidified atmosphere containing $5 \% \mathrm{CO}_{2}$ at $37^{\circ} \mathrm{C}$. The growth medium contained $440 \mathrm{ml}$ MSC basal medium,50 $\mathrm{ml}$ 10\% FBS, penicillin/streptomycin mixture. The growth medium changed every 2-3 days. The cells were passaged 3 times at approximately $80 \%$ confluence. Passage 5 BMSCs were digest and collected for determind and culture with scaffold.

\section{Characterization of BMSCs}

Flow cytometry was used to detect the MSC surface proteins such as CD29,CD44. Besides surface protein of msc, the maker of hematopoietic stem cells such as CD45,CD34 were test of the samples.

\section{Cell seeding into scaffold}

The PLGA/n-HA and gelatin/n-HA were treated by $75 \%$ achol, and then washed 3 times using phosphate buffered (PBS). BMSCs at 5 passage were suspended in growth medium at $2 \times 10^{7}$ cells $/ \mathrm{ml}$. 200ul cells suspension was droped on the top of the scaffold. The scaffold with cells was culture in cutie for 2 hours and then set in 24 well pate with growth medium. The mediu was changed every2-3 days.

\section{Animal}

60 New Zealand White rabbits (weighing 2.0-3.0kg), obtain from the animal experiments center of Anhui medical university (hefei, China). Before the experiments, approval was obtain from the Ethical Committee for animal experiments of Anhui medical university. The rabbits were anesthetized by ear vein 
of $3 \%$ pentobarbital sodium $(30 \mathrm{mg} / \mathrm{kg}$ ). Disinfection was done with iodine and $75 \%$ alcohol. A $3-5 \mathrm{~cm}$ incision was created in the middle of radius. The tissue overlying the radius were dissected. A $15 \mathrm{~mm}$ bony defect was made in the middle of radius. The forelims with $15 \mathrm{~mm}$ bone defect were immersed in seawater for 3 hours. The rabbit with seawater immersed bone defect were divided into 4 groups (group $A$, group $B$, group $C$, and group $D$ ). The group $C$ was implanted with $n-H A / P L G A / B M S C$, the group $D$ was implanted with $\beta$-TCP/PLGA/BMSCs, the groups $B$ was implanted with autograft obtained from iliac crest, the group A was implanted with anything. After implantation, the rabbits were treated with 2.4 ATA $100 \%$ HBO for 90 minutes per day for 2 weeks. The rabbits were injected intramuscularly with penicillin every day for 3 days.

\section{Radiographic examination}

Radiographs of rabbit radius were examined at 4, 8, 12 weeks after surgery under anaesthsia. Radiological evalution was done using the Lane and Sandhu scoring system ${ }^{13}$. The radiographs were evaluated by orthopedists. And the evaluation was under blinded study.

\section{Histopathologic examination}

After 4, 8, 12 weeks, the rabbits were killed by euthanasia. Samples harvested from radius defect sites were fixed in $10 \%$ neutral buffered formalin for 48 hours. The samples were decalcified with $10 \%$ EDTA solution for 30 days, dehydrated in graded ethanol and embeded in paraffin. 5 um sectons were cut and stained with hematoxylin eosin.

\section{Immunohistochemistry stain of osteocalcin}

Immunohistochemical examination of OCN was performed by the slide was deparaffinized in xylene I-II respectively for 15 minutes and dehydrayed in gradea acohol from $90 \%-70 \%$ for 3 munites. After that blocking was done with $0.5 \% \mathrm{H}_{2} \mathrm{O}_{2}$ in methanol for 30 minutes and washed by water for 5 minutes. Pertreatment of slide was performed with citrate buffer in microwave cookl and cookll for each 5 minutes followed by blocking background target to block non-specific antigens and then incubated for 15 minutes. Then it was given primary antiboby to $\mathrm{OCN}$ and incubated for 1 hour. The slide was given auniversal ink secondary antiboby to bine to the primary antiboby for 15 minutes. Conuter staining was performed with haenmtaxylin for 1-2minutes.

\section{Bone mechanical strength test}

After surgery 12 week, the mechanical strength of radius was tested by the three-point bending test. The test was performed using a universal tensile testing machine (Instron, London, UK). The ultimate force in the bending test until the specimen was broken. The bones were placed horizontally on two rounded supporting bars located at a distance of $30 \mathrm{~mm}$, and the bending load was applied at the midpoint of the defect at the loading speed of $10 \mathrm{~mm} / \mathrm{min}$ until the specimens fracture. The biomechanical properties of 
the specimens were determined by ultimate loading $(\mathrm{N})$. The data were recorded as mean plus standard error of the mean.

\section{Statistical analysis}

All data are presented as mean \pm SD. Comparisons between groups were done by one-way analysis of variance (ANOVA). The SPSS 19.0 was using for statistical analysis. The differences were considered as statistical significance at the level of $P<0.05$.

\section{Result}

\section{Flow cytometry analysis}

Flow cytometry analysis of cell surface markers showed positive expression of CD29 and CD44 and negative expression of CD34 and CD45 which displayed in figure 1.

\section{Radiological examination}

X-ray examinations were done to evaluate the development of bone regeneration in the defect, which were displayed in figure 2. At 4 weeks after surgery, litter callus formatted in group A. The shadow of autogenous bone in group $B$ was visible and callus formatted in the ends of bone defect. The shadow the area of bone defect in groups $C$ and $D$ was filled with bony callus, which was observed as the cloudy shadow. The area of cloudy shadow in group $C$ was larger than in group D. After 8 weeks the bony callus started to be absorbed, and cortical bone began to form in group $B, C$ and $D$. While the cortical bone was hard to see in group $A$. In group $B$, the autogenous bone can not be seen due to be absorbed. At 12 weeks after surgery, group $B$ exhibited significant bone formation and bony union. In the terms of bone formation and union, Group $C$ was close to group $B$, and excellent to group $D$. The defect in group $A$ was still evident, which indicated that the critical bone defect can't repaired by itself. The radiographic scoring of the x-ray was measured with the result lisited in table 1 . The assessment of repair defect demonstrated a statistically significant improve bringing of defect with $C$ compared to $D$. The group $C$ and $D$ had a higher scores than group $A(P<0.05)$, but lower than group $B(P<0.05)$.

\section{Table 1}

Lane-Sandhu radiographic scoring

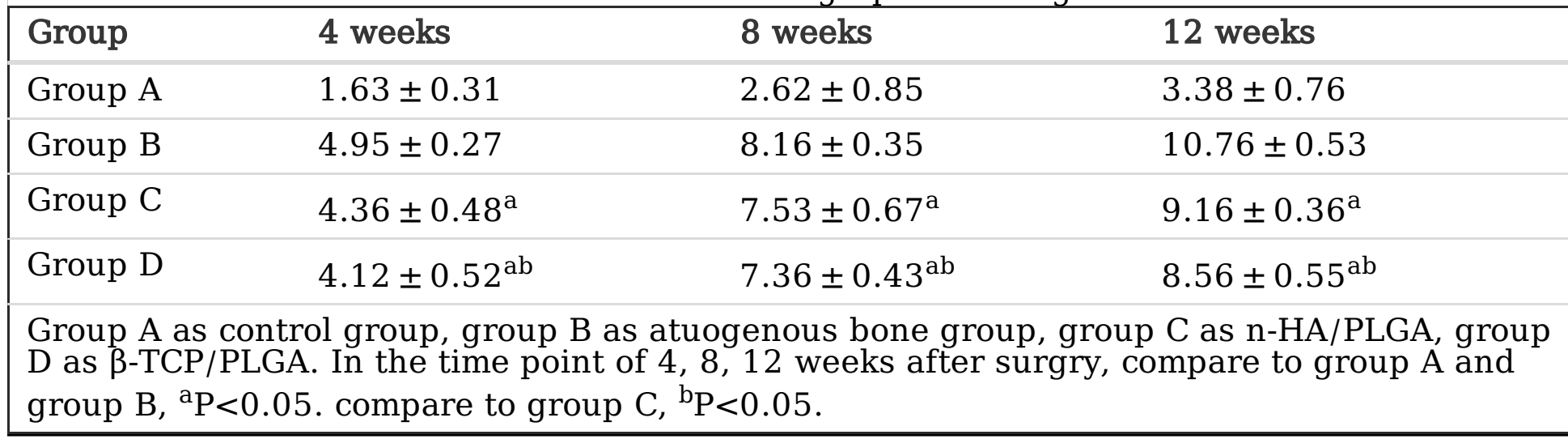




\section{Histological analysis}

The bone formation in radius defect was evaluated by $\mathrm{HE}$, which were displayed in figure 3 . The histological suopoprted the X-ray result. After 4 weeks after operation, there was no evident bone formartion in group $A$; an amount of bone-like tissue formated in defect with group $B, C$ and $D$; the scaffold in group $C$ and $D$ degraded partially. After 8 weeks, the wove bone filled the defect areas in group $B, C$ and $D$, the scaffold in group $B, C$ and $D$ degraded completely. After 12 weeks, in group A, defect without scaffold show a loose connect tissue filled in the areas. The medullary canal in group $B$ was recanalizated. Defect in group $C$ and $D$ show a larger number of wove bone formation. The new wove bone formation in defect areas in group $\mathrm{C}$ was lager than $\mathrm{D}$. There were no infection, inflammatory reaction and foreign body reaction in all groups.

\section{Immunohistochemistry analysis}

Immunohistochemical analysis was used to detect the expression of osteocalcin (OCN) within the positively stained area (brown color), during the early phase of bone repairing at 4 weeks. OCN protein expression was markedly upregulated in the defect area at 4 weeks. The expression of OCN was seen in all groups as showed in figure 4 . The result indicate that $O C N$ involved in repairment process. In addition, the brown stain intensity of $\mathrm{OCN}$ in group $\mathrm{B}, \mathrm{C}$ and $\mathrm{D}$ was denser than group $\mathrm{A}$.

\section{Biomechanical results}

The result of biomechanical testing are display in table 2 . The n-HA/PLGA and $\beta$-TCP/PLGA scaffolds enhanced the mechanical properties of the restored new bones as evidenced by a higher ultimate loading compared to the group A. However, compared to the group $B$, the biomechanics of repaired radius by nHA/PLGA and $\beta-T C P / P L G A$ were not sufficiently strong.

Table 2

biomechanical performance of 4 groups 12 weeks after surgery

\begin{tabular}{|c|c|c|c|c|}
\hline 3-point bending tese & Group A & Group B & Group C & Group D \\
\hline Ultimate strength $(\mathrm{N})$ & $51 \pm 1.6$ & $121 \pm 4.2$ & $112 \pm 5.5^{\mathrm{a}}$ & $102 \pm 2.3^{\mathrm{ab}}$ \\
\hline
\end{tabular}

\section{Discussion}

The repairment of bone defects using bone tissue engineering in animal model have been proved to be effective ${ }^{14}$. In practice, the bone defect maybe suffer from seawater immersing in naval operation. It is vital to address tissue engineering and HBO in the field of seawater immersed bone defects. Our study illuminate that the effectiveness of repairing bone defect with n-HA/PLGA /BMSCs or $\beta$ -

TCP/PLGA/BMSCs combined with HBO. 
Stem cells play a vital part in tissue engeering. In our study, allogenic bone marrow MSCs were used due to following reasons: (1) autogenic MSCs was not recommended, because surgery on the illic bone would lead to injury and pain. (2) using allogenic MSCs did't lead to immunological rejection owe to lack of expression of HLA class II antigens on MSCs. ${ }^{15}(3)$ in effectiveness of treating bone defect, there were similar between autogenic MSCs and allogenic MSCs. Soo-Hwan Kang ${ }^{16}$ compare autogenic MSC with allogenoc MSC in terms of bone regeneration in radius defect of the rabbits. On radiological, micro-CT and histologocal analysis demonstrated no evidence of immune reaction in allogenic MSCs group. Meanwhile, allogenic MSCs possess similar capacity for repairing defects compared to autologous MSCs. CD29 and CD44 were vital markers of BMSCs, BMSCs don't express CD45 and CD34. The flow cytometry ananlysis demonstrated the cells at passage 5 was still MSCs.

The reason for $\mathrm{HBO}$ improved bone healing in the study were enhancing vascularization, upregulating expression of osteogenic markers, and downregulating expression of pro-inflammatory cytokines ${ }^{17}$.

Ideal scaffolds of tiusse engineering have the characherist: good biocopatibity, appropriate degration. PLGA approved by FDA in certain clinical appliacions ${ }^{18}$, has been widely used in treatment bone defect. Whie PLGA have some disadvantages. Limited abilities of osteoconduction and osteinduction hinder using in bone tissue engineering..However, the biggest disadvantage of PLGA is its acidic degradation products, which have lower $\mathrm{pH}$ values and easily cause inflammatory reactions in the implantation area. A lower $\mathrm{pH}$ value surrounding damage the proliferation of the cells, and the inflammatory reaction can trigger the release of cytokines by the host, damaging bone formation ${ }^{19-20}$.The PLGA are acidic degradation products, $\mathrm{n}-\mathrm{HA}$ and $\beta$-TCP are alkaline degradation products ${ }^{21}$. n-HA and $\beta$-TCP can mediate the $\mathrm{pH}$ which produced by the acidic degradation products of PLGA. Therefore, they can avoid aseptic inflammation, which can provided a suitable microenvironment for new bone formation. Shu $\mathrm{He}$, et al ${ }^{20}$ provide that $\mathrm{nHA}$ can neutralize the $\mathrm{pH}$ value due to the PLGA degradation. In our study, there were no inflammation cells in $C$ and $D$ groups which indicate that n-HA/PLGA and $\beta$-TCP/PLGA have a good compatibility in the HE straning. n-HA/PLGA revealed more strengthening effects in adhesion and osteogenic differentiation of BMSCs compared to PLGA ${ }^{3}$.

A critical-sized bone defect is defined as the length of defect more than 1.5 times diameter of bone $\mathrm{e}^{22}$. The length of the radius defect was $15 \mathrm{~mm}$ for the rabbit bone defect creatded in our experiment. The length was according to the bone defect model created by $\mathrm{Yi} \mathrm{Wu}^{23}$. In the experiment of $\mathrm{Yi} W u$, the rabbits of the $15 \mathrm{~mm}$-radius defect in the control group which implanted without any materical can not repair spontaneously. In our experiment, sixty New Zealand white rabbits were created seawater immsered bone defect model in their radius. After different therapeutic measures for correspondent groups, the injured limbs were gradually recoverment. In our study, after 4 weeks of surgery, bone defect area in groups $C$ and $D$ have low density cloudy callus. After 12 weeks, there was a excellent connection and integration with broken ends, which displayed the composite of n-HA/PLGA and $\beta$-TCP/PLGA have an excellent repairment capacity for seawater immersed bone defect. There was no achievement of complete bone 
union in group $A$. The three-point bending test showed that the mechanical properties in n-HA/PLGA and $\beta$-TCP/PLGA group were closed to autograft group.

\section{Concusion}

In the experiment, the hostological and radiological study show the scaffold were degraded gradually, new callus was formed gradually, the radiographic result show that the scaffold /MSCs incorppration with HBO was successfully repair the $15 \mathrm{~mm}$ bone defect by 12 weeks after surgery. Scaffold of $\mathrm{n}-$ HA/PLGA/BMSCs and $\beta$-TCP/PLGA incorporation with HBO were effective to treat seawater immersed bone defect, and than n-HA/PLGA was more excellent than $\beta$-TCP/PLGA.

\section{Abbreviations}

BMSCs(Bone marrow mesenchymal stem cells), HBO(Hyperbaric oxygen), $\beta$-TCP (Beta tricalcium phosphate), PLGA (poly lactic-co-glycolic acid)

\section{Declarations}

\section{Ethical Approval and Consent to participate}

The experimental animal ethics committee of Anhui medical university(approval number LLSC2013031)

\section{Consent for publication}

I declare that I have obtained explicit permission from all co-authors to submit this paper in its current form

\section{Competing interests}

The authors declared no conflicts of interest.

\section{Funding}

Innovation of Science and Technology Project, Nanjing Military Region (grant no.11Z011).

\section{Authors' contributions}

Design of the work the acquisition, analysis, or interpretation of data for the work.

\section{Acknowledgements}

Thank you for the 901th Hospital of the Joint Logistics Support Force of the Chinese People's Liberation Army cell culture center for the support

Authors' information 
Gan Zhang

Orthopaedics

The 901th Hospital of the Joint Logistics Support Force of the Chinese People's Liberation Army,Hefei 230031, China

Phone:18297994598

E-mail:zhanggan10282715@163.com

\section{References}

1- Arrington ED, Smith WJ, Chambers HG, Bucknell AL, Davino NA. Complications of iliac crest bone graft harvesting. Clin Orthop Relat Res 1996 Aug;(329):300-9.

2-Faour O, Dimitriou R, Cousins CA, Giannoudis PV. The use of bone graft substitutes in large cancellous voids: any specific needs? Injury 2011 Sep;42:(Suppl 2):S87-S90.

3-Lock J, Nguyen TH, Liu H. Nanophase hydroxyapatite and poly(lactide-co-glycolide) composites promote human mesenchymal stem cell adhesionand osteogenic differentiation in vitro. J Mater Sci Mater Med. 2012 Oct;23(10):2543-52.

4-Kulkarni RK, Pani KC, Neuman C, Leonard F. Poly(lactic acid) for Surgical Implants. Arch Surg. 1966 Nov;93(5):839-43.

5-Böstman OM. Absorbable Implants for the Fixation of Fractures. J Bone Joint Surg Am. 1991 Jan;73(1):148-53.

6-Cordewener FW, Bos RR, Rozema FR, Houtman WA. Poly(L-lactide) Implants for Repair of Human Orbital Floor Defects: Clinical and Magnetic Resonance Imaging Evaluation of Long-Term Results. J Oral Maxillofac Surg. 1996 Jan;54(1):9-13.

7-Zhu H, Yang F, Tang B, Li XM, Chu YN, Liu YL, etal. Mesenchymal stem cells attenuated PLGA-induced inflammatory responses by inhibiting host DC maturation and function. Biomaterials. 2015;53:688-98.

8-Asawa Y, Sakamoto T, Komura M, Watanabe M, Nishizawa S, Takazawa Y, etal. Early stage foreign body reaction against biodegradable polymer scaffolds affects tissue regeneration during the autologous transplantation of tissue engineered cartilage in the canine model. Cell Transplant. 2012;21(7):1431-42.

9-McBane JE, Sharifpoor S, Cai K, Labow RS, Santerre JP. Biodegradation and in vivo biocompatibility of a degradable, polar/hydrophobic/ionic polyurethane for tissue engineering applications. Biomaterials. 2011 Sep;32(26):6034-44. 
10-Zong C, Xue D, Yuan W, Wang W, Shen D, Tong X, etal. Reconstruction of rat calvarial defects with mesenchymal stem cells and osteoblast-like cells in poly-lactic-co-glycolic acid scaffolds. Eur Cell Mater. 2010 Sep 1;20:109-20.

11-Jan A, Sándor GK, Brkovic BB, Peel S, Evans AW, Clokie CM. Effect of hyperbaric oxygen on grafted and nongrafted calvarial critical-sized defects. Oral Surg Oral Med Oral Pathol Oral Radiol Endod.2009 Feb;107(2):157-63.

12-Grassmann JP, Schneppendahl J, Hakimi AR, Herten M, Betsch M, Lögters TT, etal. Hyperbaric Oxygen Therapy Improves Angiogenesis and Bone Formation in Critical Sized Diaphyseal Defects. J Orthop Res. 2015 Apr;33(4):513-20.

13-Lane JM, Sandhu HS. Current approaches to experimental bone grafting. Orthop Clin North Am. 1987 Apr;18(2):213-25.

14-Wang ZX, Chen C, Zhou Q, Wang XS, Zhou G, etal. The treatment efficacy of bone tissue engineering strategy for repairing segmental bone defects under osteoporotic condition Tissue Eng Part A. 2015 Sep;21(17-18):2346-55.

15-Hee HT, Ismail HD, Lim CT, Goh JC, Wong HK. Effects of implantation of bone marrow mesenchymal stem cells, disc distraction, and combined therapy on reversing degeneration of the intervertebral disc. J Bone Joint Surg Br. 2010 May;92(5):726-36.

16-Kang SH, Chung YG, Oh IH, Kim YS, Min KO, Chung JY. Bone regeneration potential of allogeneic or autogeneic mesenchymal stem cells loaded onto cancellous bone granules in a rabbit radial defect model. Cell Tissue Res. 2014 Jan;355(1):81-8.

17-Pedersen TO, Xing Z, Finne-Wistrand A, Hellem S, Mustafa K. Hyperbaric oxygen stimulates vascularization and bone formation in rat calvarial defects. Int J Oral Maxillofac Surg. 2013 Jul;42(7):907-14.

18-Lock J, Nguyen TY, Liu H. Nanophase hydroxyapatite and poly(lactide-co-glycolide) composites promote human mesenchymal stem cell adhesion and osteogenic differentiation in vitro. J Mater Sci Mater Med. 2012 Oct;23(10):2543-52.

19-He S, Lin KF, Fan JJ, et al. Synergistic Effect of Mesoporous Silica and Hydroxyapatite in Loaded Poly(DL-lactic-co-glycolic acid) Microspheres on the Regeneration of Bone Defects. Biomed Res Int. 2016;2016:9824827.

20-Thomas MV, Puleo DA. Infection, inflammation, and bone regeneration: a paradoxical relationship. J Dent Res. 2011 Sep;90(9):1052-61.

21-Agrawal CM, Athanasion KA.Technique to control PH in vicinity of bio-degrading PLA-PLG implants. J Biomed Mater Res. 1997 Summer;38(2):105-14. 
22-Zhu ZJ, Shen H, Wang YP, Jiang Y, Zhang XL, Yuan GY. Effect of $\beta$-tricalcium phosphate/poly-l-lactide composites on radial bone defects of rabbit. Asian Pac J Trop Med. 2013 Sep;6(9):753-6.

23-Wu Y, Hou J, Yin M, Wang J, Liu C. Enhanced healing of rabbit segmental radius defects with surfacecoated calcium phosphate cement/bone morphogenetic protein-2 scaffolds Mater Sci Eng C Mater Biol Appl. 2014 Nov;44:326-35.

\section{Figures}

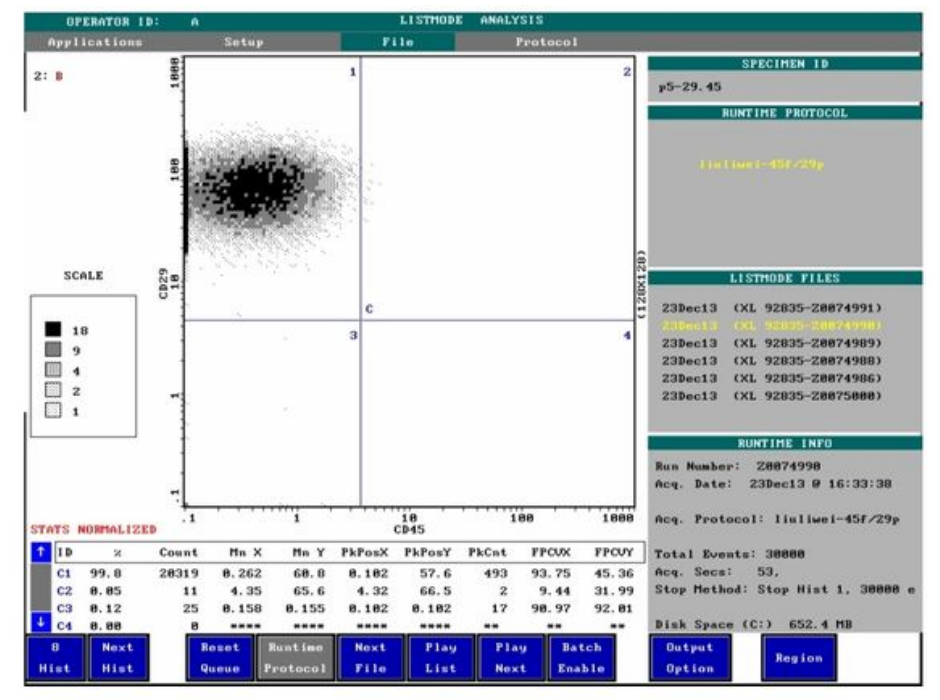

A

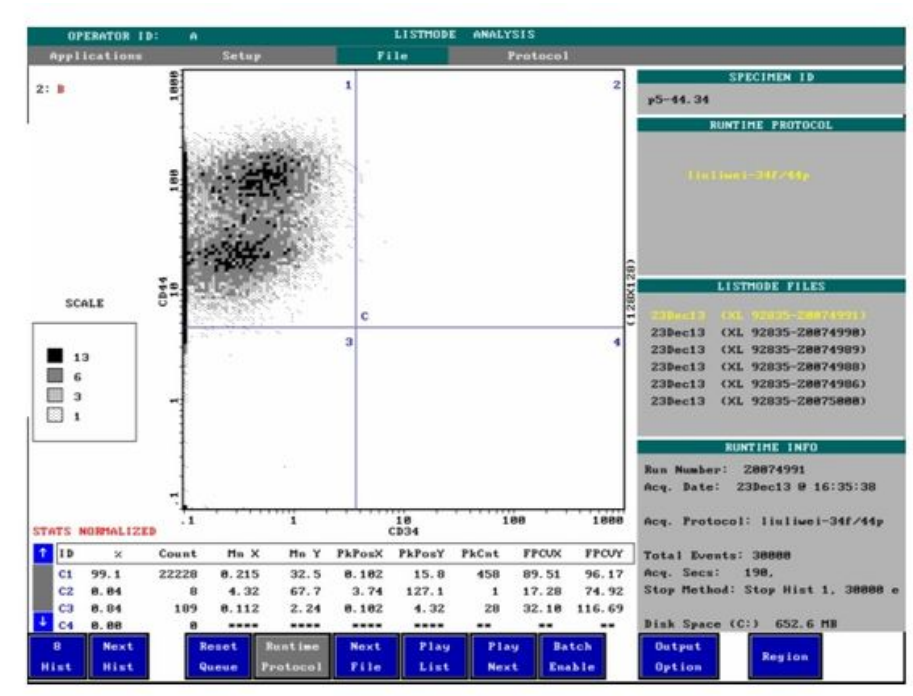

B

Figure 1

Flow cytometry analysis of cell surface markers 

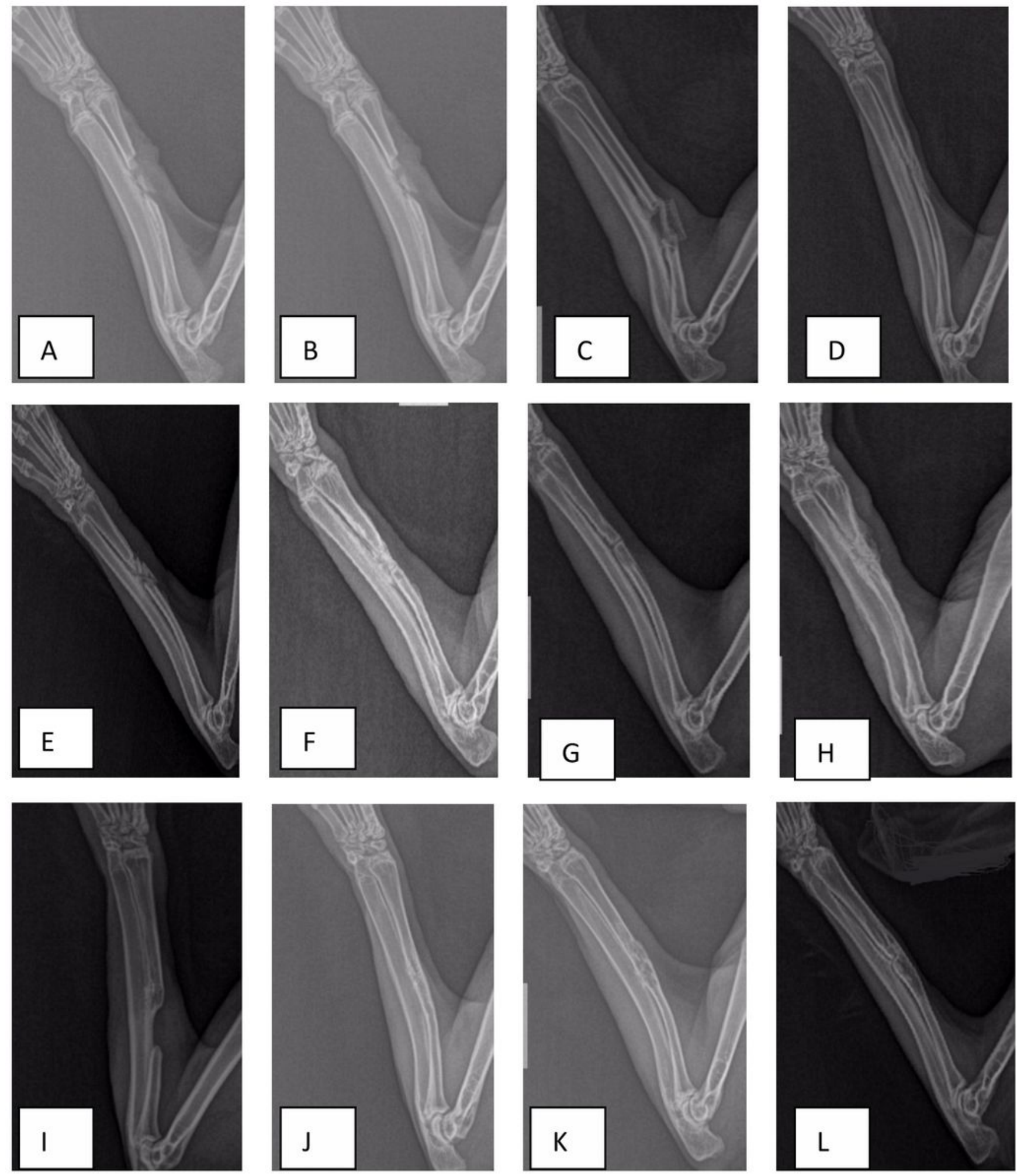

\section{Figure 2}

radiographs in A group after operation 4 weeks (A), 8 weeks (E), 12 weeks (J); B group after operation 4 weeks (B), 8 weeks (F), 12 weeks (K); C group after operation 4 weeks (C), 8 weeks (G), 12weeks囚L『; D group after operation 4 weeks (D), 8 weeks (H), 12weeks (M) 

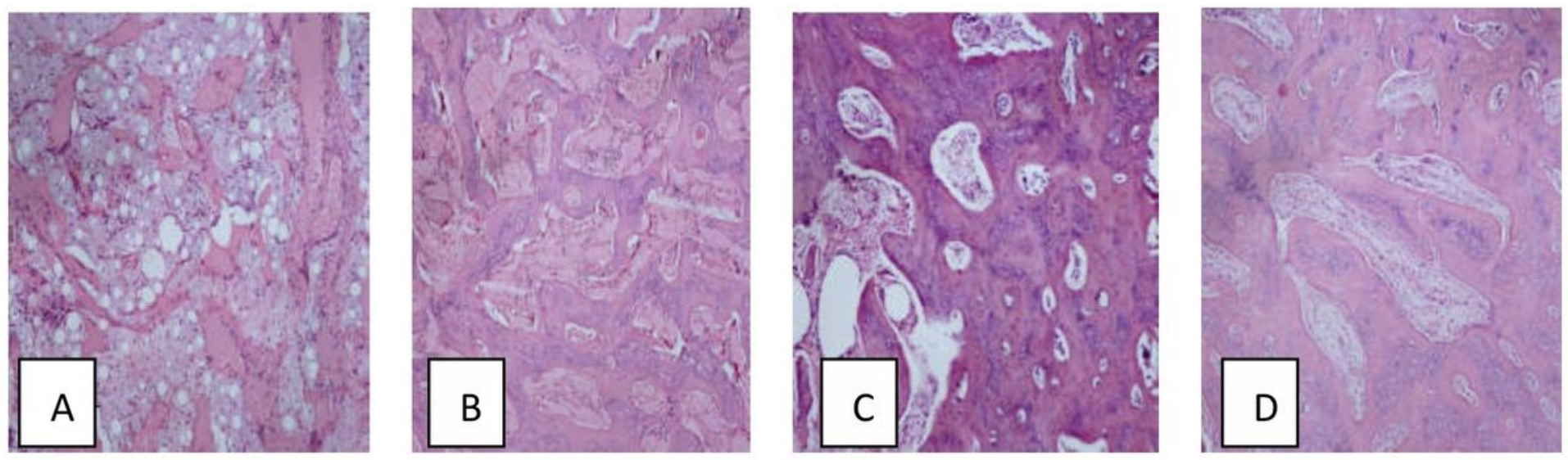

\section{Figure 3}

Hematoxylin and eosin staining for new bone tissue after operation 12 weeks
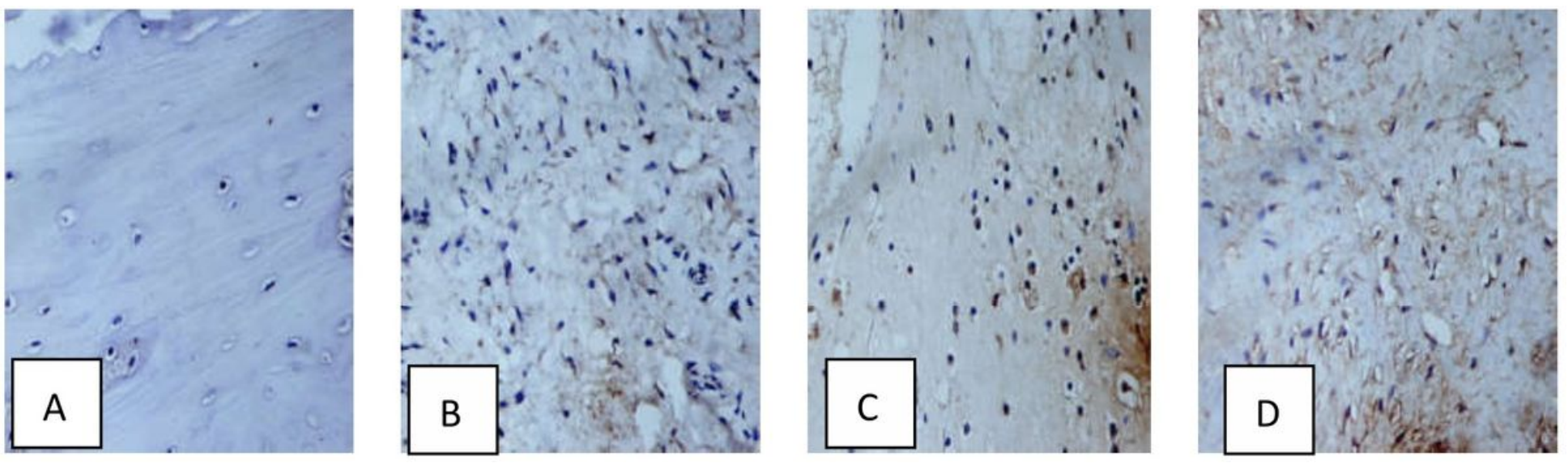

Figure 4

Immunohisochemical analysis for bone tissue after operation 4weeks 